\title{
Learning Position Controls for Hybrid Step Motors: from Current-fed to Full-Order Models
}

\author{
Valerio Salis, Nicolas Chiappinelli, Alessandro Costabeber, Pericle Zanchetta, \\ Stefano Bifaretti, Patrizio Tomei, Cristiano Maria Verrelli
}

\begin{abstract}
The experimental comparison of two different global learning position controls (namely, 'adaptive learning' and 'repetitive learning' controls) for hybrid step motors performing repetitive tasks has been recently presented in the literature. Related benefits and drawbacks have been successfully analyzed on the same robotic application. However, the design of the two aforementioned learning controls - though relying on a rigorous stability analysis - are based on a simplified current-fed model of the motor. They cannot achieve precise current tracking due to the mere presence of PI control actions in the outer current control loops. The aim of this paper is to enrich and update the results of the above comparison in the light of the latest contributions that generalize the theoretical design to the fullorder voltage-fed motor models of hybrid step motors. Learning actions are now included in the outer current control loops: they generalize the corresponding PI actions to the periodic scenario and allow to solve a control problem whose solution was seeming very difficult to be obtained.
\end{abstract}

Index Terms-Permanent magnet step motors, learning control, position tracking.

\section{INTRODUCTION}

Stepper motors are electro-mechanical devices that convert 'electrical pulses' into discrete mechanical movements. One among the most significant advantages of step motors (accuracy and repeatability, high efficiency, power density and high torque to inertia ratio, excellent durability and serviceability, absence of external rotor excitation and windings, excellent response to starting/stopping/reversing commands) is the ability to be controlled in an open loop fashion. Position is known simply by keeping track of the input step pulses. One relevant type of step motors is the hybrid one: it simultaneously combines the features of both the permanent magnet and variable reluctance type step motors. The rotor is multi-toothed (like the variable reluctance type one) and contains an axially magnetized concentric magnet around its shaft. The teeth on the rotor provide a suitable path that guides the magnetic flux to preferred locations in the air-gap. This in turn increases the detent, holding, dynamic torque characteristics of the motor. However, when one step pulse is applied to the step motor (especially when moving in a large step increment)

N. Chiappinelli and S. Bifaretti are with the Industrial Engineering Department, University of Rome 'Tor Vergata', Via del Politecnico 1, 00133 Roma, Italy.

V. Salis, A. Costabeber, P. Zanchetta are with the Electrical \& Electronic Engineering Department, University of Nottingham, University Park, NG7 2RD, Nottingham, UK.

P. Tomei and C.M. Verrelli are with the Electronic Engineering Department, University of Rome 'Tor Vergata', Via del Politecnico 1, 00133 Roma, Italy, (e-mail (corresponding author): verrelli@ing.uniroma2.it). overshoots and ringing can arise. This is certainly undesirable in applications in which high-precision position tracking is required. Furthermore, step motors can often exhibit resonance behaviours at certain step rates [1]. Compensation of torque pulsations by feedback actions thus constitutes an attractive solution [2]-[8]. The non-uniformity in the developed torque due to the non-sinusoidal flux distribution in the air-gap [9] which causes speed oscillations and deteriorates the system performance especially at low speeds - is however to be explicitly taken into account in the control design in order to achieve high-precision position tracking.

With this respect, advanced learning control techniques are actually able to take into account such non-uniformity [10][12]. The only constraint is to consider position reference profiles that are periodic signals with known period. Learning controls aim in fact at performing a system inversion by reconstructing the unknown input reference corresponding to the periodic position reference. Therefore, in contrast to standard model-based robust adaptive techniques [13] or to standard adaptive or extended-state observer-based controls [14]-[16], they require neither high gains in the inner speed/position control loops nor restriction of model uncertainties to be modelled by finite-dimensional linear or nonlinear exo-systems with known dimension. In this context, two main approaches to learning control can be adopted, which require neither torque transducers nor resetting procedures' ${ }^{1}$ i) the 'adaptive learning' approach in [12]; ii) the 'repetitive/iterative learning' approach in [11], [17]-[19]. While the adaptive learning approach consists in interpreting the uncertain reference input as an uncertain periodic signal with known period whose finite Fourier expansion is to be estimated ${ }^{2}$, the repetitive learning approach uses the input recorded during the previous trial, in

\footnotetext{
${ }^{1}$ Resetting procedures are typically required by standard iterative learning controls in quite a number of applications involving robotic mechanisms, batch reactors and assembly lines.

${ }^{2}$ The periodic time function $\pi(t)$ (with period $T_{*}$ ) is identified by developing it in Fourier series as

$$
\pi(t)=\sum_{l=0}^{+\infty} \sigma_{l} \varphi_{l}(t)
$$

and by estimating the $M$ (constant) coefficients of its finite Fourier expansion

$$
\sum_{l=0}^{M-1} \sigma_{l} \varphi_{l}(t) \doteq \sigma[M]^{T} \Phi_{M}(t)
$$

where: $\sigma[M]=\left[\sigma_{0}, \ldots, \sigma_{M-1}\right]^{T}, \Phi_{M}(t)=\left[\varphi_{0}(t), \ldots, \varphi_{M-1}(t)\right]^{T}$, $\varphi_{0}(t)=1, \varphi_{2 j}(t)=\sqrt{2} \cos \left(j t \frac{2 \pi}{T_{*}}\right), \varphi_{2 j-1}(t)=\sqrt{2} \sin \left(j t \frac{2 \pi}{T_{*}}\right)$ $(j=1, \ldots,(M-1) / 2)$.
} 
conjunction with the run-time tracking error, to generate the input signal to be exerted in each trial. The successful use of such approaches in position tracking control of hybrid step motors has been however limited to the restrictive current-fed model. In the experimental comparison in [10], benefits and drawbacks of the two learning approach are in fact analyzed on the same robotic application by just including PI control actions in the outer current control loops. Such PI control actions cannot achieve precise current tracking. The reasons for this theoretical limitation were constituted by the several technical difficulties arising when the full-order voltage-fed model of the hybrid step motor is considered in the stability analysis:

- an uncertain function multiplies the rotor speed derivative in the motor model;

- unstructured uncertainties appear in the current dynamics.

Such technical difficulties have been recently solved in [11] and [12]. In particular:

- the global results achieved in the current-fed case become semi-global in the voltage-fed one;

- the resulting innovative control design and stability analysis necessarily involve more than one learning estimation scheme;

- learning actions - being crucially included even in the outer current control loops - generalize the PI actions to the periodic scenario and allow for convergence to zero of the current tracking errors.

The aim of this paper is to enrich and update the results of the experimental comparison in [10] in the light of the aforementioned contributions in [11] and [12] (just merely containing simulation results). The motor application described in [10] is again used for the comparison: a metal bar link connected to the rotor shaft with a brass ball at the free end - reproducing the behaviour of a position-dependent single-link robotic load - is required to track a known periodic position reference. Experimentally applying to the same specific real problem the latest theoretical advances in learning position control theory for full-order model step motors allows us to quantitatively and qualitatively extend the comparison in [10] by studying in detail the effects of different features and requirements in a rather complex scenario.

\section{DyNAMIC MODEL}

Assume that both the stator self inductance variations with position and the mutual inductance between stator windings are negligible, whereas the non-sinusoidal flux distribution in the air-gap is modeled by the higher order harmonics in the mutual inductance terms (between the $\mathrm{j}^{\text {th }}$ phase and the fictitious rotor winding). The full-order dynamics of a hybrid step motor with two phases in the $(d, q)$ reference frame rotating at speed $N_{r} \omega$ and identified by the angle $N_{r} \theta$ in the fixed $(a, b)$ reference frame attached to the stator $(\theta$ is the rotor position, $\omega$ is the rotor speed and $N_{r}$ is the number of rotor teeth) are then given by [11] (see [13] for model derivation):

$$
\begin{aligned}
\frac{\mathrm{d} \theta(t)}{\mathrm{d} t}= & \omega(t) \\
h_{p}(\theta(t)) \frac{\mathrm{d} \omega(t)}{\mathrm{d} t}= & -\alpha_{p}(\theta(t))-\beta_{p}(\theta(t)) \omega(t) \\
& +c_{p}(\theta(t)) i_{d}(t)+i_{q}(t) \\
\frac{\mathrm{d} i_{d}(t)}{\mathrm{d} t}= & -\frac{R}{L_{0}} i_{d}(t)+N_{r} i_{q}(t) \omega(t) \\
& -\frac{\omega(t)}{L_{0}} \eta_{d}(\theta(t))+\frac{1}{L_{0}} u_{d}(t) \\
\frac{\mathrm{d} i_{q}(t)}{\mathrm{d} t}= & -\frac{R}{L_{0}} i_{q}(t)-N_{r} i_{d}(t) \omega(t) \\
& -\frac{\omega(t)}{L_{0}} \eta_{q}(\theta(t))+\frac{1}{L_{0}} u_{q}(t)
\end{aligned}
$$

where

$$
\begin{aligned}
& h_{p}(\theta)=\frac{J}{i_{f} N_{r}}\left[\sum_{j=1}^{n} j L_{m j} \cos \left[(1-j) N_{r} \theta\right]\right]^{-1} \\
& \alpha_{p}(\theta)=\frac{h_{p}(\theta)}{J}\left[T_{L}(\theta)+\frac{N_{r} i_{f}^{2}}{2} \sum_{j=4}^{n} j L_{f j} \sin \left[j N_{r} \theta\right]\right] \\
& \beta_{p}(\theta)=\frac{D h_{p}(\theta)}{J} \\
& c_{p}(\theta)=\frac{h_{p}(\theta) i_{f} N_{r}}{J} \sum_{j=2}^{n} j L_{m j} \sin \left[(1-j) N_{r} \theta\right] \\
& \eta_{d}(\theta)=-i_{f} N_{r} \sum_{j=2}^{n} j L_{m j} \sin \left[(j-1) N_{r} \theta\right] \\
& \eta_{q}(\theta)=i_{f} N_{r} \sum_{j=1}^{n} j L_{m j} \cos \left[(j-1) N_{r} \theta\right]
\end{aligned}
$$

and: $\left(i_{d}, i_{q}\right)$ are the stator current vector $(d, q)$ components; $\left(u_{d}, u_{q}\right)$ are the stator voltage vector $(d, q)$ components; $n \geq 4$ is a positive integer; $D$ is the friction coefficient; $J$ is the rotor inertia; $T_{L}(\cdot)$ is the load torque; $i_{f}$ is the fictitious rotor current provided by the permanent magnet; $R$ and $L_{0}$ are the stator windings resistance and the self inductance, respectively; the harmonics $\sum_{j=1}^{n} L_{m j} \cos \left[j N_{r} \theta\right]$ and $\sum_{j=1}^{n} L_{m j} \cos \left[j N_{r} \theta-\frac{\pi}{2}\right]$ model the non-sinusoidal flux distribution in the air-gap; the term $\frac{N_{r} i_{f}^{2}}{2} \sum_{j=4}^{n} j L_{f j} \sin \left[j N_{r} \theta\right]$ represents the disturbance torque due to cogging; the parameters $L_{m j}, 2 \leq j \leq n$ (which are zero under the standard assumption of sinusoidal flux distribution) are much smaller ${ }^{3}$ than $L_{m 1}$. All the (constant) system parameters (including the number $n$ of harmonics) along with the load torque function are here allowed to be uncertain excepting for the number of rotor teeth $N_{r}$ and the stator windings self inductance $L_{0}$.

\section{EQUiVALENT PERIODIC DISTURBANCES}

Since a non-zero $i_{d}$ only contributes to torque ripples, it is desirable to set the $i_{d}$-reference $i_{d}^{*}=0$, while choosing

\footnotetext{
${ }^{3}$ The direct-axis current $i_{d}$ does not thus significantly contribute to torque production, whereas the quadrature-axis current $i_{q}$ is assigned to produce the required torque.
} 
the $i_{q}$-reference $i_{q}^{*}$ to produce the desired torque reference. Define the (back-stepping-based) rotor speed reference ( $k_{\theta}$ is a positive control parameter):

$$
\omega^{*}=-k_{\theta} e_{\theta}+\dot{\theta}^{*}
$$

leading to the rotor position and speed tracking errors:

$$
\begin{aligned}
& e_{\theta}=\theta-\theta^{*} \\
& e_{\omega}=\omega-\omega^{*} \doteq \omega+k_{\theta} e_{\theta}-\dot{\theta}^{*} .
\end{aligned}
$$

Accordingly obtain the rotor position error dynamics:

$$
\dot{e}_{\theta}=-k_{\theta} e_{\theta}+e_{\omega} .
$$

Then express the uncertain function

$$
f_{c}(\theta, \omega)=\alpha_{p}(\theta)+\beta_{p}(\theta) \omega
$$

as

$$
\begin{aligned}
f_{c}(\theta, \omega)= & q_{0 c}\left(\theta^{*}, \dot{\theta}^{*}, \ddot{\theta}^{*}\right)-h_{p}(\theta) \ddot{\theta}^{*}+g_{c}\left(e_{\theta}, e_{\omega}, t\right) \\
& +h_{p}(\theta) k_{\theta} e_{\omega}-h_{p}(\theta) k_{\theta}^{2} e_{\theta}
\end{aligned}
$$

with

$$
\begin{aligned}
q_{0 c}\left(\theta^{*}, \dot{\theta}^{*}, \ddot{\theta}^{*}\right)= & \alpha_{p}\left(\theta^{*}\right)+\beta_{p}\left(\theta^{*}\right) \dot{\theta}^{*}+h_{p}\left(\theta^{*}\right) \ddot{\theta}^{*} \\
g_{c}\left(e_{\theta}, e_{\omega}, t\right)= & -h_{p}(\theta) k_{\theta} e_{\omega}+h_{p}(\theta) k_{\theta}^{2} e_{\theta} \\
& +\alpha_{p}(\theta)-\alpha_{p}\left(\theta^{*}\right)+\beta_{p}\left(\theta^{*}\right)\left(e_{\omega}-k_{\theta} e_{\theta}\right) \\
& +\left[\beta_{p}(\theta)-\beta_{p}\left(\theta^{*}\right)\right] \omega \\
& +\left[h_{p}(\theta)-h_{p}\left(\theta^{*}\right)\right] \ddot{\theta}^{*} .
\end{aligned}
$$

Due to the assumption that the position reference signal is $T_{*}$-periodic,

$$
q_{c}(t)=q_{0 c}\left(\theta^{*}(t), \dot{\theta}^{*}(t), \ddot{\theta}^{*}(t)\right)
$$

is a periodic function with known period $T_{*}$. It constitutes the uncertain periodic input reference for the current $i_{q}(t)$ that achieves perfect tracking for $i_{d}=0$ and for compatible initial conditions $\theta(0)=\theta^{*}(0), \omega(0)=\dot{\theta}^{*}(0)$. Similarly, introduce the functions $q_{d}(t)$ and $q_{q}(t)$ defined as

$$
\begin{aligned}
& q_{d}(t)=\dot{\theta}^{*}(t) \eta_{d}\left(\theta^{*}(t)\right) \\
& q_{q}(t)=\dot{\theta}^{*}(t) \eta_{q}\left(\theta^{*}(t)\right)+R q_{c}(t),
\end{aligned}
$$

which affect the stator current dynamics and are also periodic with known period $T_{*}$.

\section{THE TWO LATEST LEARNING CONTROLS}

In this paper we report the state feedback learning controls in [11] and [12] for system (1). They guarantee rotor position tracking of a reference signal $\theta^{*}(t)$, which is assumed to belong to the following class:

- $\theta^{*}(t)$ is a sufficiently smooth periodic function with known period $T_{*}$ (i.e. $\theta^{*}(t)=\theta^{*}\left(t+T_{*}\right), \forall t \geq-T_{*}$ ), with bounded time derivatives $\theta^{*(i)}(t)(i=1,2)$ for all $t \in\left[0, T_{*}\right)$.

Such learning control algorithms ${ }^{4}$ - with the terms characterizing the adaptive learning control to appear as overbraces read:

\footnotetext{
${ }^{4}$ Robustifying terms in [11], [12] are neglected along with certain nonnecessary $\left(e_{\theta}, e_{\omega}\right)$-feedback terms in the definition of $u_{q}$ that are however used, for the sake of completeness, in the experiments.
}

i) the inner rotor position/speed control loop

$$
\begin{aligned}
i_{q}^{*}(t) & =-k_{\omega} e_{\omega}(t)-k_{v} e_{\theta}(t)+\overbrace{\operatorname{sat}_{B_{q}}\left(\hat{q}_{c}\left(t-T_{*}\right)\right)}^{\hat{\rho}[N]^{\mathrm{T}}(t) \Phi_{N}(t)} \\
e_{\theta} & =\theta-\theta^{*}, \quad e_{\omega}=\omega+k_{\theta} e_{\theta}-\dot{\theta}^{*}
\end{aligned}
$$

ii) the outer stator current vector control loop

$$
\begin{aligned}
u_{d}(t)= & L_{0}\left[-N_{r} \omega(t) i_{q}(t)-\frac{k_{i d}}{r_{d}} e_{i, d}(t)\right] \\
& +\overbrace{\hat{q}_{d}(t)}^{\hat{\alpha}\left[M_{d}\right]^{\mathrm{T}}(t) \Phi_{M_{d}}(t)} \\
u_{q}(t)= & L_{0}\left[N_{r} \omega(t) i_{d}(t)-\frac{k_{i q}}{r_{q}} e_{i, q}(t)\right. \\
& +\overbrace{\frac{\mathrm{d}}{\mathrm{d} t} \operatorname{sat}_{B_{q}}\left(\hat{q}_{c}\left(t-T_{*}\right)\right)}^{\frac{\mathrm{d}}{\mathrm{d}}[N]^{\mathrm{T}}(t) \Phi_{N}(t)}] \\
& +\overbrace{\hat{\delta}\left[M_{q}\right]^{\mathrm{T}}(t) \Phi_{M_{q}}(t)}
\end{aligned}
$$

in which $e_{i, d}=i_{d}-i_{d}^{*}=i_{d}, e_{i, q}=i_{q}-i_{q}^{*}$ are the stator current tracking errors.

The estimates $\hat{q}_{c}(t), \hat{q}_{d}(t), \hat{q}_{c}(t)$ of the periodic functions $q_{c}(t)$, $q_{d}(t), q_{q}(t)$ are provided by the repetitive learning estimation schemes:

$$
\begin{aligned}
\hat{q}_{c}(t) & =\operatorname{sat}_{B_{q}}\left(\hat{q}_{c}\left(t-T_{*}\right)\right)-\mu_{q} \varphi_{T_{*}}(t) \mathcal{F}_{q}(t) \\
\hat{q}_{c}(t) & =0, \forall t \leq 0 \\
\mathcal{F}_{q}(t) & =e_{\omega}(t)+r_{q} \operatorname{sgn}\left[b_{c}(\theta(t))\right] e_{i, q}(t) \\
b_{c}(\theta(t)) & =k_{\omega} L_{0}-R h_{p}(\theta(t))
\end{aligned}
$$

and

$$
\begin{aligned}
& \hat{q}_{d}(t)=\operatorname{sat}_{B_{q d}}\left(\hat{q}_{d}\left(t-T_{*}\right)\right)-\mu_{\alpha} \varphi_{T_{*}}(t) e_{i, d}(t) \\
& \hat{q}_{d}(t)=0, \forall t \leq 0 \\
& \hat{q}_{q}(t)=\operatorname{sat}_{B_{q q}}\left(\hat{q}_{q}\left(t-T_{*}\right)\right)-\mu_{\delta} \varphi_{T_{*}}(t) e_{i, q}(t) \\
& \hat{q}_{q}(t)=0, \forall t \leq 0
\end{aligned}
$$

in the repetitive learning control and by the adaptive learning estimation schemes:

$$
\begin{aligned}
\dot{\hat{\rho}}[N] & =\operatorname{Proj}\left[-\mu_{q} \Phi_{N} e_{\omega}, \hat{\rho}[N], \nu_{\rho}, B_{q}\right] \\
\|\hat{\rho}[N](0)\| & \leq \frac{B_{q}}{\sqrt{N}}
\end{aligned}
$$

and $^{5}$

$$
\begin{aligned}
\dot{\hat{i}}_{d} & =N_{r} \omega i_{q}+\frac{1}{L_{0}} u_{d}-\frac{\hat{\alpha}\left[M_{d}\right]^{T} \Phi_{M_{d}}}{L_{0}}+k_{e d} e_{e, d} \\
\dot{\hat{i}}_{q} & =-N_{r} \omega i_{d}+\frac{1}{L_{0}} u_{q}-\frac{\hat{\delta}\left[M_{q}\right]^{T} \Phi_{M_{q}}}{L_{0}}+k_{e q} e_{e, q} \\
\dot{\hat{\alpha}}\left[M_{d}\right] & =\operatorname{Proj}\left[-\mu_{\alpha} \frac{\Phi_{M_{d}}}{L_{0}} e_{e, d}, \hat{\alpha}\left[M_{d}\right], \nu_{\alpha}, B_{q d}\right] \\
\left\|\hat{\alpha}\left[M_{d}\right](0)\right\| & \leq B_{q d}
\end{aligned}
$$

${ }^{5}$ The subsequent adaptive estimation scheme resort to $\left(\hat{i}_{d}, \hat{i}_{q}\right)$ as auxiliary variables. 


$$
\begin{aligned}
\dot{\hat{\delta}}\left[M_{q}\right] & =\operatorname{Proj}\left[-\mu_{\delta} \frac{\Phi_{M_{q}}}{L_{0}} e_{e, q}, \hat{\delta}\left[M_{q}\right], \nu_{\delta}, B_{q q}\right] \\
\left\|\hat{\delta}\left[M_{q}\right](0)\right\| & \leq B_{q q} \\
e_{e, d} & =i_{d}-\hat{i}_{d}, \quad e_{e, q}=i_{q}-\hat{i}_{q}
\end{aligned}
$$

in the adaptive learning control. The above control algorithms depend on:

- the positive control parameters $k_{\omega}$ (chosen in the repetitive learning control to guarantee that $b_{c}(\theta(t))$ is different from zero for any $t \geq 0)$ and $k_{v}, k_{\theta}, r_{d}, r_{q}, k_{i d}, k_{i q}, \mu_{q}$, $\mu_{\alpha}, \mu_{\delta}$

- the known positive bounds $B_{q}, B_{q d}, B_{q q}$ on $\left|q_{c}(t)\right|$, $\left|q_{d}(t)\right|,\left|q_{q}(t)\right|$, respectively;

- the saturation function $\operatorname{sat}_{M_{\xi}}(\cdot): \mathbb{R} \rightarrow\left[-M_{\xi}-\delta_{s}, M_{\xi}+\right.$ $\left.\delta_{s}\right]$, which is a class $\mathcal{C}^{1}$ odd increasing function satisfying ( $\delta_{s}$ is an arbitrary positive real) $\operatorname{sat}_{M_{\xi}}(q)=q$ for any $q \in\left(0, M_{\xi}\right], \lim _{q \rightarrow \infty} \operatorname{sat}_{M_{\xi}}(q)=M_{\xi}+\delta_{s}$ and $\left|q_{1}-q_{2}\right| \geq$ $\left|q_{1}-\operatorname{sat}_{M_{\xi}}\left(q_{2}\right)\right|$ for any $\left|q_{1}\right| \leq M_{\xi}, q_{2} \in \mathbb{R}$;

- the function $\varphi_{x}(\cdot): \mathbb{R}_{0}^{+} \rightarrow[0,1](x>0)$, which is a class $\mathcal{C}^{1}$ increasing function for $t \in[0, x]$ (with $\varphi_{x}(0)=\dot{\varphi}_{x}(0)=0, \dot{\varphi}_{x}(x)=0, \varphi_{x}(t)=1$ for any $t \geq x$ ) endowing the above estimates with suitable continuity properties (in accordance with the expressions (12)-(13) when time $t$ approaches zero);

- the classical projection algorithm $\operatorname{Proj}\left[\xi_{z}, \hat{z}, \nu_{z}, B_{z}\right]$ in [12], which is used to modify the adaptation law $\dot{\hat{z}}=\xi_{z}$ into $\dot{\hat{z}}=\operatorname{Proj}\left[\xi_{z}, \hat{z}, \nu_{z}, B_{z}\right]$ in order to constrain, for any $t \geq 0$, the vector estimate $\hat{z}(t)$ into the ball with center at the origin and radius equal to $\nu_{z}+B_{z}\left(\nu_{z}>0\right)$.

\section{Comparative theoretical remarks}

- The strategy adopted by both the proposed learning controls consists in compensating torque pulsations by feedback actions in order to achieve high precision position tracking ${ }^{6}$.

- Both the proposed learning controllers feed back the signal:

$-k_{\omega} e_{\omega}(t)-k_{v} e_{\theta}(t)=-\left(k_{v}+k_{\omega} k_{\theta}\right) e_{\theta}(t)-k_{\omega} \dot{e}_{\theta}(t)$,

which corresponds to a PD action on the rotor position tracking error being able to stabilize the $\left(e_{\theta}, \dot{e}_{\theta}\right)$-second order system in companion-like form. They also include the plug-in signals $\operatorname{sat}_{B_{q}}\left(\hat{q}_{c}\left(t-T_{*}\right)\right)$ or $\hat{\rho}[N]^{\mathrm{T}}(t) \Phi_{N}(t)$, which generalize to the periodic scenario the integral action $-k_{i} \int_{0}^{t} e_{\theta}(\tau) \mathrm{d} \tau$ that is typically used to compensate the constant disturbances affecting the rotor speed error dynamics.

- The learning estimation schemes in the outer stator current control loop replace the integral actions on the stator current tracking errors. The repetitive learning estimation schemes in fact reduce - for $T_{*} \rightarrow 0^{7}$ - to the classical integral actions when saturation and $\varphi_{T_{*}}$ functions are

\footnotetext{
${ }^{6}$ This does not necessarily imply torque ripple reduction.

${ }^{7} \mathrm{~A}$ constant signal is a periodic signal characterized by any arbitrarily small period, so that a repetitive learning estimation scheme with any arbitrarily small real as period can be successfully adopted to learn a constant signal (see [20]).
}

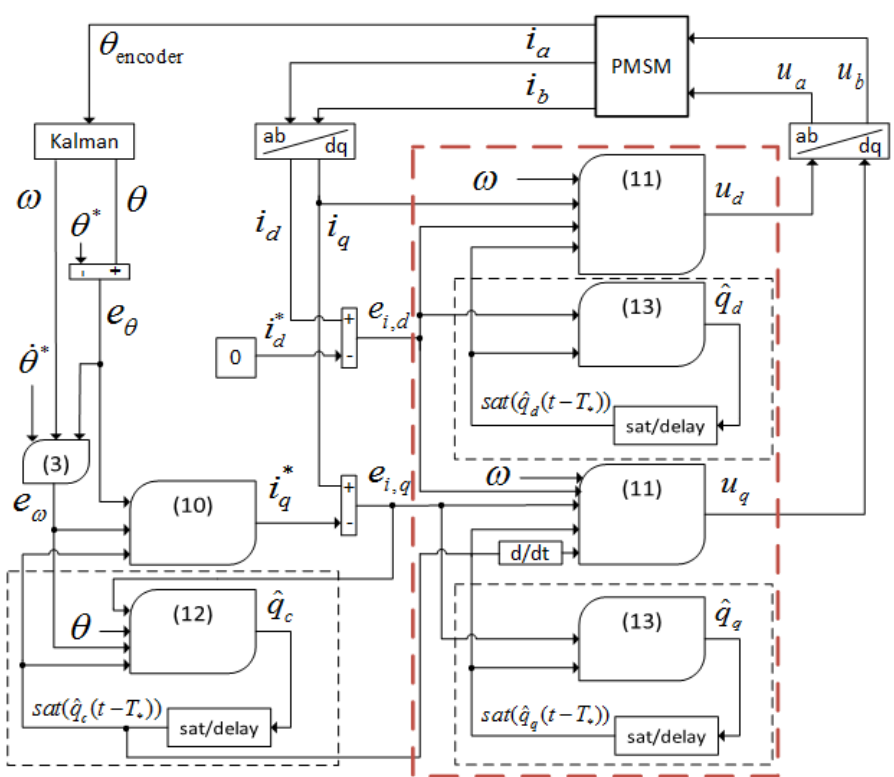

Fig. 1. Block diagram describing the repetitive learning control scheme in (2)-(3), (10)-(13).

neglected and the learning gains are multiplied by $T_{*}$. The adaptive learning estimation schemes, on the other hand, reduce to the classical integral actions when the projection algorithm is neglected and only $\varphi_{0}$ is considered.

Figures 1 and 2 report the block diagrams for both the learning controllers presented in this section. They describe the structure of the proposed control schemes and highlight the improvements with respect to the previous solutions derived for current-fed motors. In particular, the content of red dashed boxes - describing the outer current control loops - replaces the PI control actions of [10] (see also [19]).

\section{Tuning issues}

The above learning controls rely on design parameters to be tuned.

- The control parameters $k_{v}, k_{\theta}, k_{\omega}$ characterize the PD feedback action on the the position tracking error $e_{\theta}(t)$. In particular, the parameter $k_{\theta}$ characterizes the $e_{\theta}(t)$ feedback term in the back-stepping definition of the reference $\omega^{*}(t)=-k_{\theta} e_{\theta}+\dot{\theta}^{*}$ in (10), while the parameter $k_{\omega}$ plays an analogous role in the definition of $i_{q}^{*}$ in (10). The larger such parameters are chosen, the stronger the corresponding feedback actions on the $\left(e_{\theta}(t), e_{\omega}(t)\right)$ error dynamics result.

- The parameters $\mu_{q}, \mu_{\alpha}, \mu_{\delta}$ are the learning gains in (12)-(13) and (14)-(15). The larger such parameters are chosen, the larger the influence of the correction terms on the corresponding estimate behaviour results. They play a role that extends to the periodic case the one played by the integral action gains. 


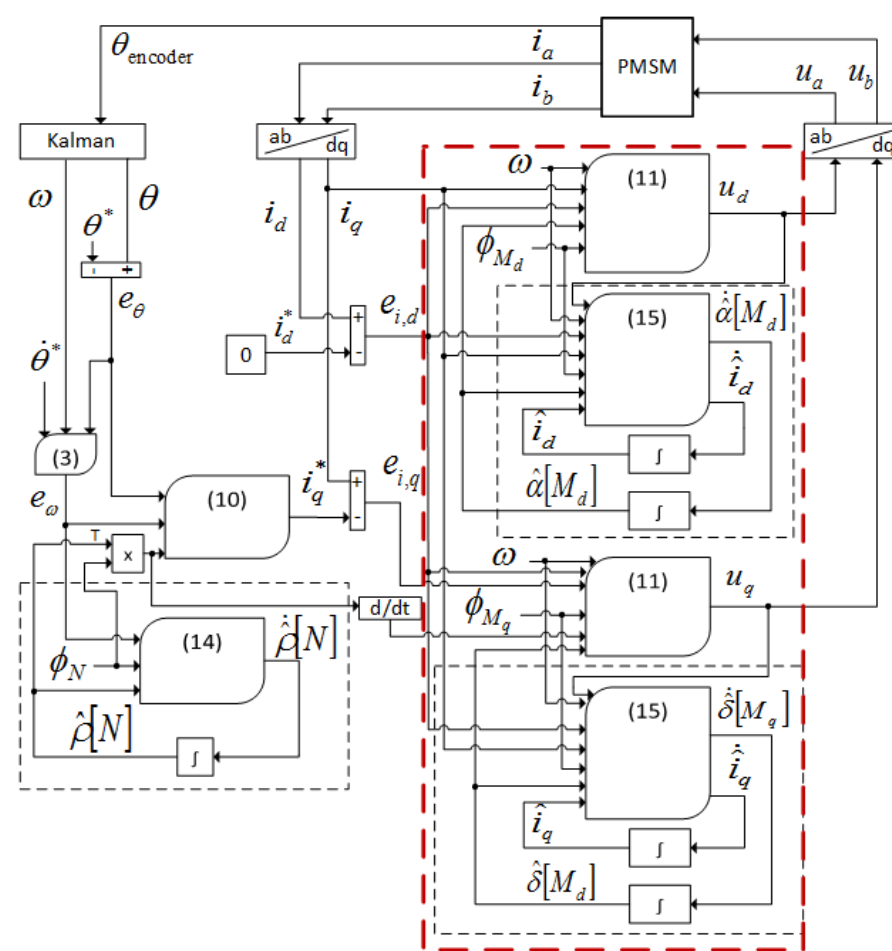

Fig. 2. Block diagram describing the adaptive learning control scheme in (2)-(3), (10)-(11), (14)-(15).

\section{Padé approximants for the repetitive learning control}

When the delay terms are approximated as in [17] by properly using the Padé rational functions (see Remark 3 and stability/convergence implications therein), we obtain learning estimation schemes with inputs $\mathcal{F}_{q}, e_{i, d}, e_{i, q}$ and outputs $\hat{q}_{c}, \hat{q}_{d}, \hat{q}_{q}$, respectively, which are linear and boundedinput bounded-output (the saturation functions along with the $\varphi_{T_{*}}(\cdot)$ - functions are no longer required). Here, no first order filters are involved (so that $\alpha=0$ in [17]), while, for the sake of clarity, the same Padé approximants with odd order $m$ are used for the three learning estimation schemes (see [17] for comments on the role of $m$ and [18] for a relevant robotic application). In particular, the resulting control is guaranteed to be bounded and, when compared to (10)-(13), involves ( $s$ is the complex variable):

$$
\begin{aligned}
\Lambda(s) & =\frac{\beta}{1+\beta}\left(\mathcal{P}_{[m, m]}(s)+1\right) \hat{Q}_{c}(s) \\
M(s) & =s \Lambda(s)
\end{aligned}
$$

in place of $\operatorname{sat}_{B_{q}}\left(\hat{q}_{c}\left(t-T_{*}\right)\right)$ and its time derivative $\frac{\mathrm{d}}{\mathrm{d} t} \operatorname{sat}_{B_{q}}\left(\hat{q}_{c}\left(t-T_{*}\right)\right)$ in (10)-(11), respectively, with the modified linear learning estimation schemes in place of (12)-(13) ${ }^{8}$ :

$$
\begin{aligned}
& \hat{Q}_{c}(s)=-\frac{d_{p}(s)}{q_{\pi}(s)} \mu_{q} F_{q}(s) \\
& \hat{Q}_{d}(s)=-\frac{d_{p}(s)}{q_{\pi}(s)} \mu_{\alpha} E_{i, d}(s)
\end{aligned}
$$

\footnotetext{
${ }^{8} \hat{Q}_{c}(s), \hat{Q}_{d}(s), \hat{Q}_{q}(s), F_{q}(s), E_{i, d}(s), E_{i, q}(s)$ are the Laplace transforms of the Laplace transformable signals $\hat{q}_{c}(t), \hat{q}_{d}(t), \hat{q}_{q}(t), \mathcal{F}_{q}(t), e_{i, d}(t)$, $e_{i, q}(t)$, respectively.
}

$$
\begin{gathered}
\hat{Q}_{q}(s)=-\frac{d_{p}(s)}{q_{\pi}(s)} \mu_{\delta} E_{i, q}(s) \\
\text { incorporating the }[m, m] \text {-Padé approximant of } \mathrm{e}^{-s T_{*}} \text { : } \\
\mathcal{P}_{[m, m]}(s)=\frac{P_{m}\left(-s T_{*}\right)}{P_{m}\left(s T_{*}\right)} \doteq \frac{n_{p}(s)}{d_{p}(s)}
\end{gathered}
$$

Here $P_{m}\left(s T_{*}\right)$ denotes $\sum_{k=0}^{m}\left(\begin{array}{c}m \\ k\end{array}\right) \frac{(2 m-k) !}{(2 m) !}\left(s T_{*}\right)^{k}$, while $\beta<1$ is such that $q_{\pi}(s)=d_{p}(s)-\beta n_{p}(s)$ has all its roots in $\mathbb{C}^{-}$. It is relevant to notice that, for $\mu_{q}=\bar{\mu}_{q} T_{*}, \mu_{\alpha}=\bar{\mu}_{\alpha} T_{*}$, $\mu_{\delta}=\bar{\mu}_{\delta} T_{*}, \beta=1$, we get:

$$
\begin{aligned}
& T_{*}{ }^{-1}\left(1-\mathcal{P}_{[m, m]}(s)\right) \hat{Q}_{c}(s)=-\bar{\mu}_{q} F_{q}(s) \\
& T_{*}{ }^{-1}\left(1-\mathcal{P}_{[m, m]}(s)\right) \hat{Q}_{d}(s)=-\bar{\mu}_{\alpha} E_{i, d}(s) \\
& T_{*}{ }^{-1}\left(1-\mathcal{P}_{[m, m]}(s)\right) \hat{Q}_{q}(s)=-\bar{\mu}_{\delta} E_{i, q}(s)
\end{aligned}
$$

with the limit of the previous left-hand-sides for $T_{*} \rightarrow 0$ being equal to $s \hat{Q}_{c}(s), s \hat{Q}_{d}(s)$ and $s \hat{Q}_{q}(s)$, respectively and $\Lambda(s)$ converging to $\hat{Q}_{c}(s)$. In other words, for $T_{*} \rightarrow 0$ (corresponding to the case of constant references $\theta^{*}$ ), even the three above Padé-based learning estimation schemes reduce to simple integral actions (see the related comparative remarks).

\section{THEORETICAL DISCUSSION}

We shall compare in the next experimental section the adaptive learning control with the non-infinite-memory (stabilized) version of the repetitive learning control. While the key-idea of approximating infinite-dimensional exo-systems by finitedimensional ones is implicitly underlying the adaptive learning control approach, the finite-dimensional approximation of $\mathrm{e}^{-s T_{*}}$ by the $[m, m]$-Padé approximant $\mathcal{P}_{[m, m]}(s T)$ takes place at a different design level, that is after the control design and not before it. The resulting non-infinite-memory approximations of the repetitive estimation schemes constitute linear time-invariant finite-dimensional dynamic systems: they are described by transfer functions exhibiting all their poles with negative real part $^{9}$, so that typical long term instability issues of classical repetitive learning controls due to high frequency disturbance noises are avoided. In digital implementations, the continuous-time state differential equations of the realization for the proper rational function $\mathrm{e}^{-s T_{*}}$ (approximating the $T_{*}$ delay line in the repetitive learning estimation scheme) are approximated by discrete-time state difference equations. Such difference equations involve the storage, in the case of first order Euler approximations, of a number of values equal to the corresponding dimension of the state and a computational cost that grows proportionally to the above dimension. The dimension of the state grows with the Padé approximation order $m$, while playing a role similar to the one played by the number of stored values at each trial in [10]. The larger $m$ is (exactly like the number of stored values at each trial in [10] or the number $M$ of sinusoidal basis functions in the adaptive learning approach described in footnote 2), the smaller is the approximation error in reproducing the internal

\footnotetext{
${ }^{9}$ This is in contrast to repetitive estimation schemes that use a finite number of stored values (see [10]) and that would have here required high-order polynomials to approximate the delay-signal.
} 
model by the corresponding control scheme. Again, the digital implementation of the Padé-based linear repetitive learning estimation scheme represents - for any $m$ - a discrete-time approximation of an 'exponentially stable' continuous-time dynamic repetitive learning estimation scheme with learning capabilities. This is in contrast to the digital implementation of the adaptive learning control (or of the learning estimation scheme using a finite number of stored values in [10]). Such 'exponentially stable' nature, which is related to the choice of $\beta<1$ and leads to a bounded-input, bounded-output learning estimation law without the use of saturation functions, is the theoretical counterpart of the possible inclusion - in place of the projection algorithm - of a forgetting term in the adaptive estimation scheme of the adaptive learning control.

\section{EXPERIMENTAL SET UP AND RESULTS}

In this section, both the learning position controls are experimentally tested and compared on the same robotic application. The rotor position reference signal is chosen as the output ${ }^{10}$ of the linear third order filter

$$
\begin{aligned}
& \dot{\zeta}_{1}=\zeta_{2} \\
& \dot{\zeta}_{2}=\zeta_{3} \\
& \dot{\zeta}_{3}=-1728 \zeta_{1}-432 \zeta_{2}-36 \zeta_{3}+w_{\zeta} \\
& y_{\zeta}=1728 \zeta_{1}
\end{aligned}
$$

whose input $w_{\zeta}=\theta^{*}(t)=1.2 \sin (\pi t / 2)$ is periodic with period $T=4 \mathrm{~s}$.

In the experiments, the initial conditions have been set to $\theta(0)=\pi$ and $\omega(0)=0$. This choice ${ }^{11}$ complies with a realistic setting in which the motor is initially at rest and the initial position is compatible with the corresponding reference signal. In order to make significant the comparison, the same values for the common design parameters $k_{\theta}=13, k_{\omega}=5, k_{v}=-2$, $k_{i d}=k_{i q}=9, r_{d}=r_{q}=L_{0}$ are chosen, while

$$
\operatorname{RMS}_{e_{\theta},[32,36] \mathrm{s}}=\sqrt{\frac{1}{4} \int_{32}^{36} e_{\theta}(\tau)^{2} \mathrm{~d} \tau}
$$

is taken, from [10], as performance index.

\section{A. The experimental set up}

The Aerotech 310SMB3 two-phase hybrid step motor, manufactured by Eastern Air Devices Inc., is used in the experiments (see Table I for its main properties). The rotor shaft is connected to a metal bar link (21 cm of length) with a brass ball at the end.

As shown in the functional block diagram of Fig. 3, the motor is fed by two H-bridges (one for each phase).

The DC bus voltage is generated by TDK-LambdaGEN300-11 Rack Programmable PSU set to $80 \mathrm{~V}_{\mathrm{dc}}$ and 10 $\mathrm{A}$ as max-current, with an RC filter acting on the output.

\footnotetext{
${ }^{10}$ The robustness of the proposed control algorithms with respect to nonperiodic disturbances affecting the rotor position reference signal is thus tested.

${ }^{11}$ The rotor angle $\theta=\pi$ corresponds to the vertical position of the robotic load.
}

\begin{tabular}{|c|c|}
\hline Stall torque & $2.6 \mathrm{Nm}$ \\
\hline Rated voltage & $80 \mathrm{~V} \mathrm{DC}$ \\
\hline Rated phase current & $6 \mathrm{~A}$ \\
\hline Maximal radial load & $156 \mathrm{~N}$ \\
\hline Maximal thrust load & $267 \mathrm{~N}$ \\
\hline
\end{tabular}

TABLE I

MAIN MOTOR PROPERTIES.

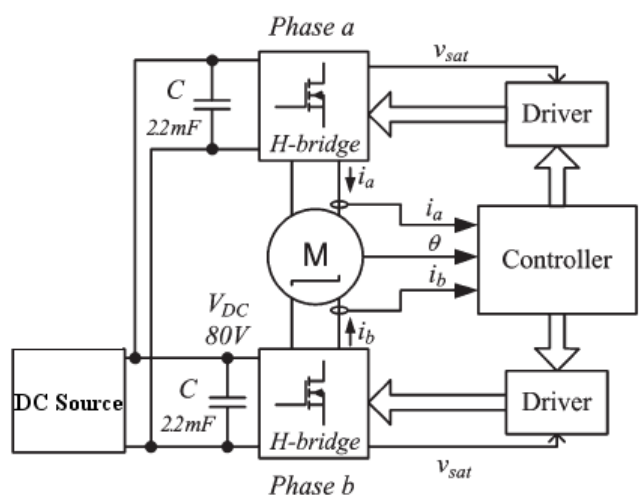

Fig. 3. Functional block diagram.

The experimental tests have been performed by applying a $4 \mathrm{kHz}$ switching frequency- PWM signal to the power inverters (FSBB20CH60C-3-phase-inverter) used as H-bridges. A Texas Instruments controller board, based on the DSP TMS320F28335, has been employed to implement the proposed control algorithms and to generate the logic driving signals for the power switches (see Figs. 3 and 4). Each control algorithm has been executed with a sampling interval $T_{s}=250 \mu \mathrm{s}$. At the beginning of each sampling interval, the phase currents values (provided by two Hall effect current sensors) have been acquired along with the rotor position. The rotor position measurement has been provided by a 1000 pulse per revolution encoder interfaced to a dedicated hardware unit on the DSP that counts the rising and falling edges of the two quadrature encoder signals. In order to reduce the speed noise due to the relatively low encoder resolution, the discrete-time Kalman filter (with a $20 \mathrm{~Hz}$ cut-off frequency) proposed in [21] has been used, whose choice is motivated by the rather low phase lag. The experimental prototype is shown in detail in Fig. 4, with the main mechanical and electronic subsystems being highlighted by dashed boxes.

\section{B. The adaptive learning control}

The remaining control parameters for the adaptive learning control (all values are in SI units) are: $\mu_{q}=1, \mu_{\alpha}=0.6$, $\mu_{\delta}=0.6, N=M_{d}=M_{q}=15$, while $\hat{\rho}[N](0)=\hat{\alpha}[N](0)=$ $\hat{\delta}[N](0)=0$. Fig. 5 shows the rotor position tracking error $e_{\theta}(t)$, while Fig. 6 shows the time histories of $\omega(t), e_{\omega}(t)$, $\hat{\rho}[N]^{\mathrm{T}}(t) \Phi_{N}(t), i_{q}^{*}(t)$. The stator currents $\left(i_{a}(t), i_{b}(t)\right)$, which are captured on the oscilloscope, are reported in Fig. 7. The $(d, q)$ stator currents tracking errors $e_{i, d}(t), e_{i, q}(t)$ are also finally reported in Fig. 8.

In order to illustrate the benefits of estimating the above (large) number of Fourier coefficients, the same experiment is 


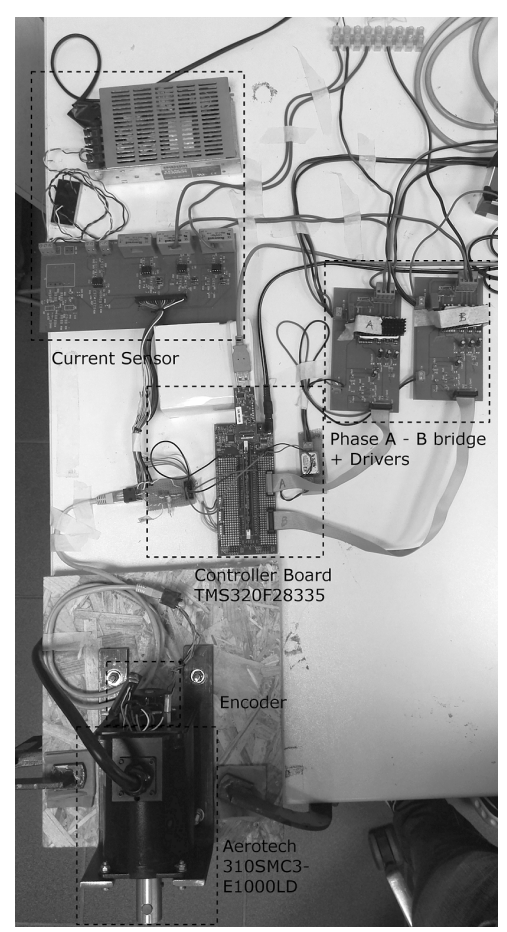

Fig. 4. Experimental prototype (top-view photograph).

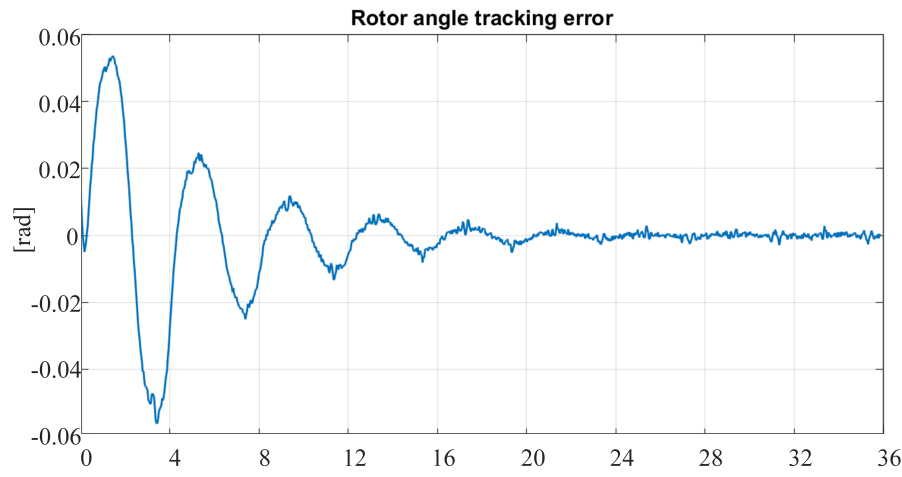

Fig. 5. Adaptive learning control with $N \stackrel{[\mathrm{s}]}{=} M_{d}=M_{q}=15$ : rotor position tracking error $e_{\theta}(t)$.
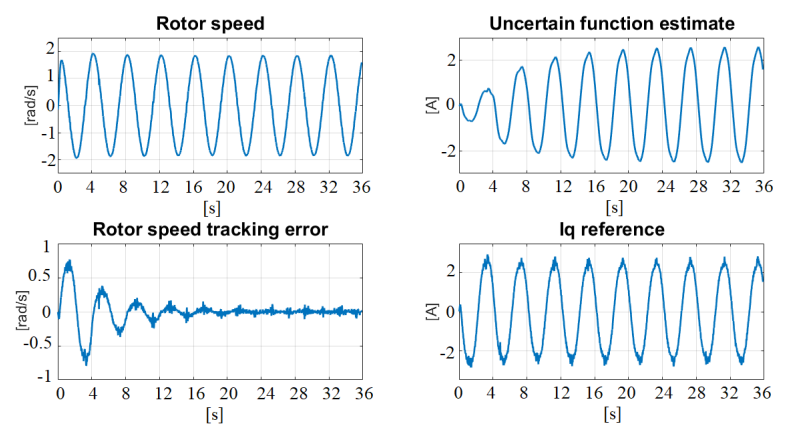

Fig. 6. Adaptive learning control with $N=M_{d}=M_{q}=15: \omega(t), e_{\omega}(t)$, $\hat{\rho}[N]^{\mathrm{T}}(t) \Phi_{N}(t), i_{q}^{*}(t)$.

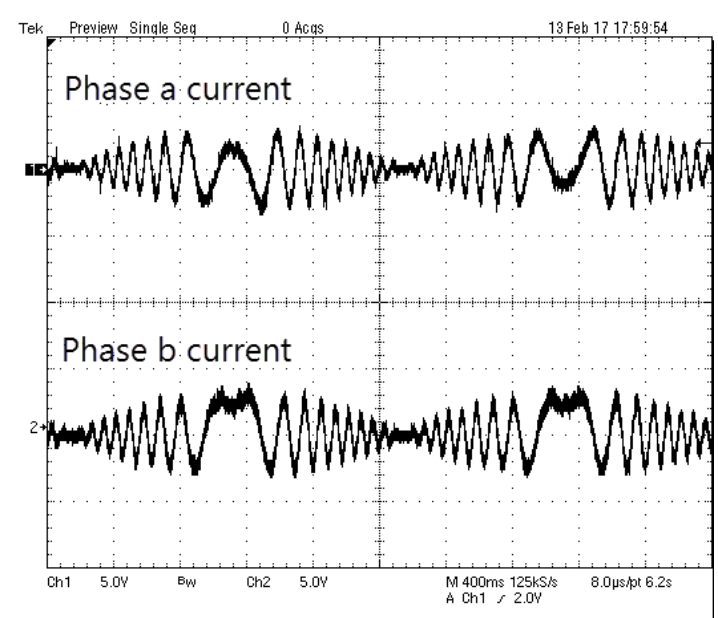

Fig. 7. Adaptive learning control with $N=M_{d}=M_{q}=15$ : stator currents $\left(i_{a}(t), i_{b}(t)\right)$.
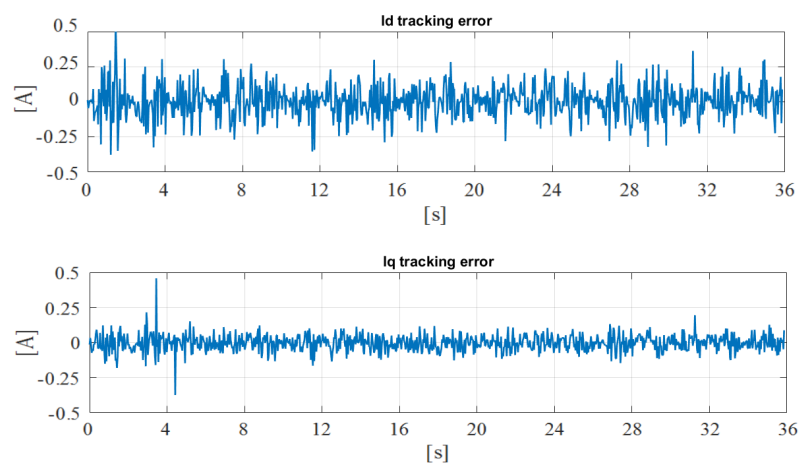

Fig. 8. Adaptive learning control with $N=M_{d}=M_{q}=15$ : $(d, q)$ stator currents tracking errors $e_{i, d}(t), e_{i, q}(t)$.

carried out with $N=M_{d}=M_{q}=5$ instead of $N=M_{d}=$ $M_{q}=15$ : as shown in Fig. 9, in which $e_{\theta}(t)$ is reported, a larger residual tracking error is obtained (see Table II for the resulting performance index values).

The $(d, q)$ stator currents tracking errors $e_{i, d}(t), e_{i, q}(t)$ are reported in Fig. 10 for $N=M_{d}=M_{q}=5$.

\section{The Padé-based repetitive learning control}

The remaining control parameters for the Padé-based repetitive learning control (all values are in SI units) are: $\mu_{q}=14$, $\mu_{\alpha}=6, \mu_{\delta}=6$, while $m=7$. The Tustin transformation $z=\frac{1-T_{s} / 2}{1+T_{s} / 2}$ has been applied to the Padé $s$-functions in order to translate them into the $z$-domain, while preserving the stability of the control. The algebraic loop induced by this transformation has been directly removed in the $\mathrm{C}$ -

\begin{tabular}{|c|c|}
\hline$N=M_{d}=M_{q}$ & Performance index $\mathrm{RMS}_{e_{\theta},[32,36] \mathrm{s}}$ \\
\hline 5 & $2.4 \cdot 10^{-3}$ \\
\hline 15 & $0.795 \cdot 10^{-3}$ \\
\hline
\end{tabular}

TABLE II

PERFORMANCE INDEX VALUES FOR THE ADAPTIVE LEARNING CONTROL. 


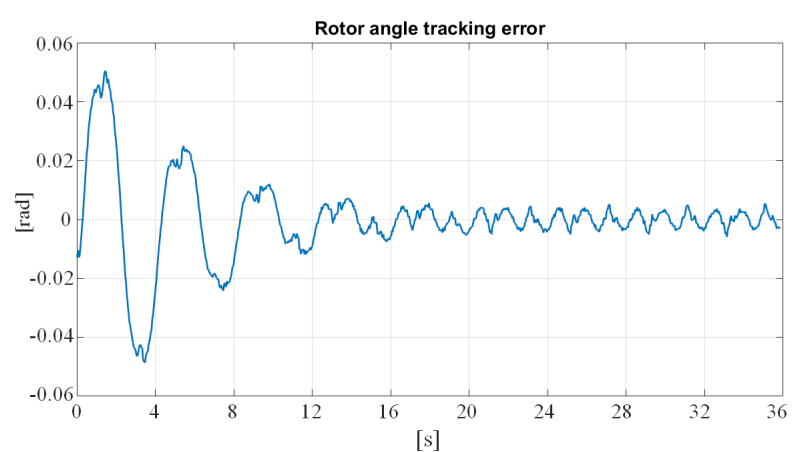

Fig. 9. Adaptive learning control with $N=M_{d}=M_{q}=5$ : rotor position tracking error $e_{\theta}(t)$.
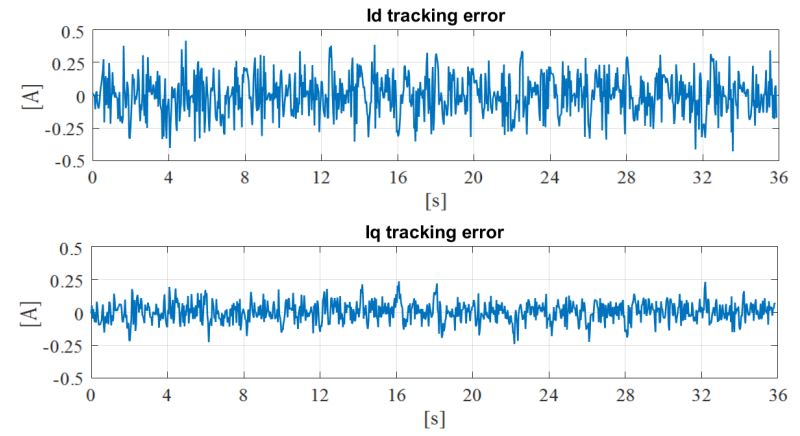

Fig. 10. Adaptive learning control with $N=M_{d}=M_{q}=5:(d, q)$ stator currents tracking errors $e_{i, d}(t), e_{i, q}(t)$.

language implementation ${ }^{12}$. Fig. 11 shows the rotor position tracking error $e_{\theta}(t)$, while Fig. 12 shows the time histories of $\omega(t), e_{\omega}(t), \lambda(t)$ (inverse Laplace transform of $\Lambda(s)$ ), $i_{q}^{*}(t)$. The stator currents $\left(i_{a}(t), i_{b}(t)\right)$, which are captured on the oscilloscope, are reported in Fig. 13. The $(d, q)$ stator currents tracking errors $e_{i, d}(t), e_{i, q}(t)$ are finally reported in Fig. 14.

In order to illustrate the benefits of using a large $m$, the same experiment is carried out with $m=3$ instead of $m=7$ : as shown in Fig. 15, in which $e_{\theta}(t)$ is reported, a larger residual tracking error is obtained (see Table III for the resulting

\footnotetext{
${ }^{12}$ Such algebraic loop is due to the fact that the discrete state-space model associated to $\Lambda(s)$ includes a rather small direct link between the output $i_{q}^{*}(k)$ and the input $\mathcal{F}_{q}(k)$, which in turn again depends on $i_{q}^{*}(k)$. Such rather small dependency has been removed in the implementation process.
}

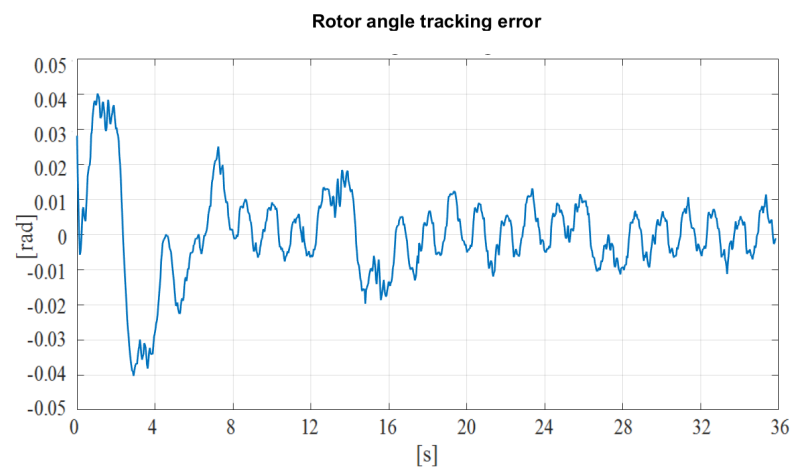

Fig. 11. Padé-based repetitive learning control with $m=7$ : rotor position tracking error $e_{\theta}(t)$.
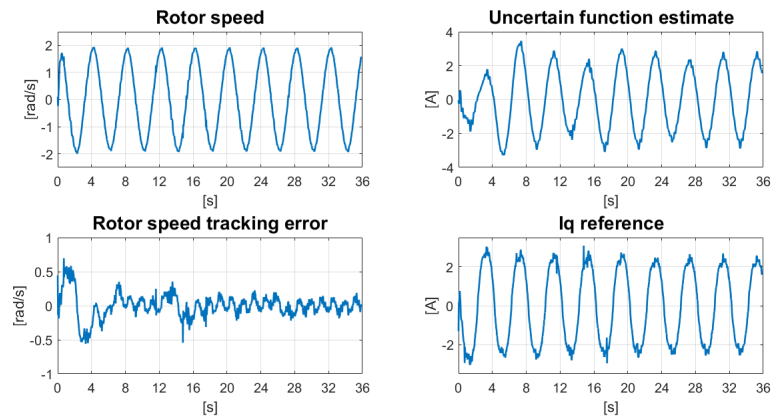

lq reference

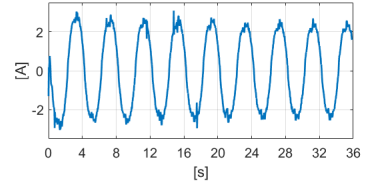

Fig. 12. Padé-based repetitive learning control with $m=7: \omega(t), e_{\omega}(t)$, $\lambda(t), i_{q}^{*}(t)$.

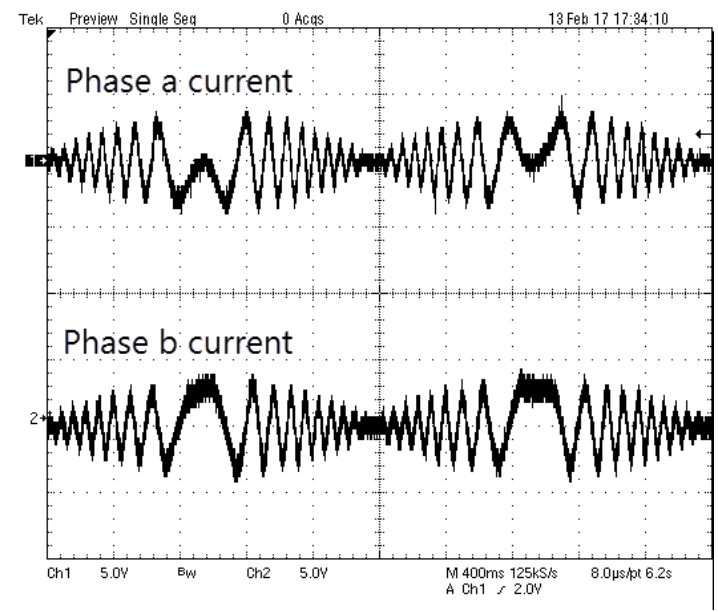

Fig. 13. Padé-based repetitive learning control with $m=7$ : stator currents $\left(i_{a}(t), i_{b}(t)\right)$.

performance index values).

The $(d, q)$ stator currents tracking errors $e_{i, d}(t), e_{i, q}(t)$ are also reported in Fig. 16 for $m=3$.

\section{EXPERIMENTAL DISCUSSION}

Comparative experimental considerations are in order:

- in contrast to the results in [10], here the closed loop behaviour in the first trial $(t \leq 4 \mathrm{~s})$ is no longer worst for the repetitive learning position control: the fact that no learning action is performed for $t \leq T_{*}$ (characterizing the finite-memory version of the repetitive estimation scheme in [10]) is not here inherited from the Padé-based repetitive learning scheme;

- in accordance with [10], similar steady-state residual tracking errors are obtained by both the learning position controls: small values of $m$ for the Padé-based repetitive

\begin{tabular}{|c|c|}
\hline$m$ & Performance index $\mathrm{RMS}_{e_{\theta},[32,36] \mathrm{s}}$ \\
\hline 3 & $14.6 \cdot 10^{-3}$ \\
\hline 7 & $5 \cdot 10^{-3}$ \\
\hline
\end{tabular}

TABLE III

PERFormanCE INDEX VALUES FOR THE PADÉ-BASED REPETITIVE LEARNING CONTROL. 
Id tracking error

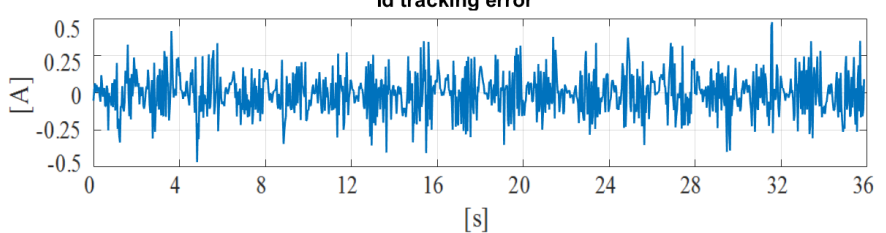

lq tracking error

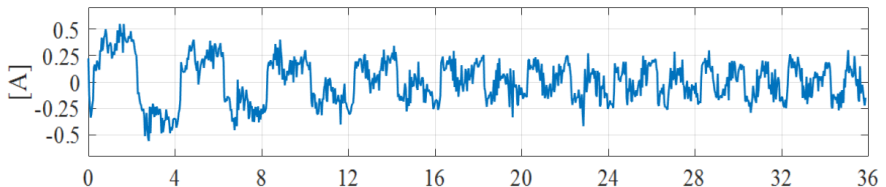

Fig. 14. Padé-based repetitive learning [spntrol with $m=7:(d, q)$ stator currents tracking errors $e_{i, d}(t), e_{i, q}(t)$.

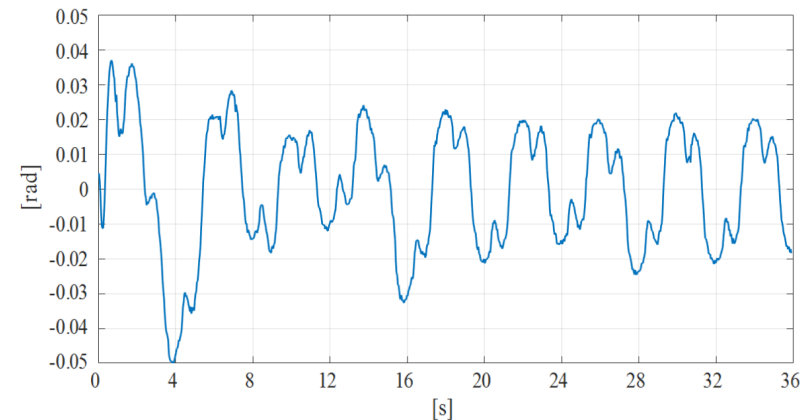

Fig. 15. Padé-based repetitive learning control with $m=3$ : rotor position tracking error $e_{\theta}(t)$.

learning control do not however guarantee comparably satisfactory tracking capabilities (even due to the stabilizing action performed by $\beta<1$ );

- in contrast to the results in [10], in which the stator current tracking errors exhibit always the same magnitude (due to the mere presence of PI control actions), here the learning estimation schemes in the outer current control loops allow increasingly satisfactory stator current tracking for larger $N=M_{d}=M_{q}$ or $m$;

- in contrast to [10], here the uncertain function estimate $\lambda(t)$ in $i_{q}^{*}(t)$ of the Padé-based repetitive learning control filters the measurement errors in $e_{\omega}(t)$ : this is related to the presence of the delayed term $\operatorname{sat}_{B_{q}}\left(\hat{q}_{c}\left(t-T_{*}\right)\right)$ in
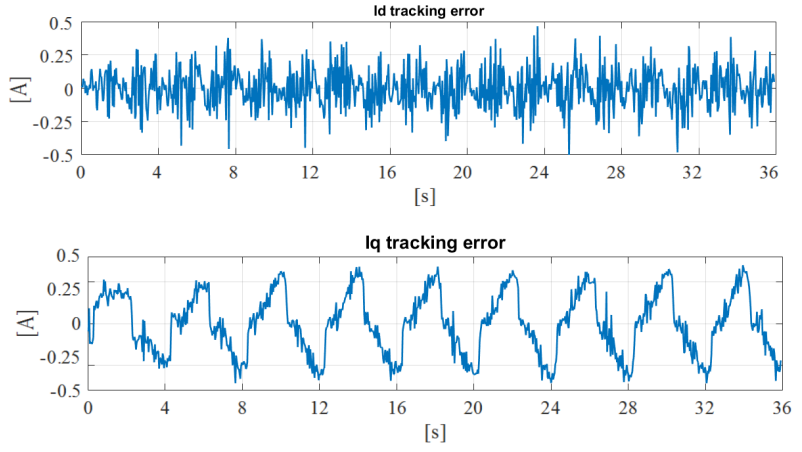

Fig. 16. Padé-based repetitive learning control with $m=3:(d, q)$ stator currents tracking errors $e_{i, d}(t), e_{i, q}(t)$. place of the non-delayed corresponding term in [10] and leads to better rotor speed behaviours;

- while in the adaptive learning control the sinusoidal basis functions are externally generated, in the Padé-based repetitive learning control the 'internal model' is directly included in the controller, so that some additional computational efforts concerning the Tustin transformation and the related algorithm implementation in C-language are to be taken into account.

\section{CONCLUSIONS}

Both the adaptive and the repetitive learning position controls recently designed in [11] and [12] for voltage-fed hybrid step motors have been, for the first time, experimentally tested and compared with reference to the same robotic application described in [10]. Simple structure and small number of design parameters to be tuned, as well as absence of resetting procedures, keep on being definite advantages for both the presented controllers. Simulation results in [11] and [12] are successfully confirmed by the presented experiments in the presence of unmodeled dynamics, discrete-time controller implementation, encoder quantization errors, as well as rotor speed filtering and current sensing. The role played on the tracking performance by the number of estimated Fourier coefficients in the adaptive learning control is the same played by the approximation order in the Padé-based repetitive learning control.

\section{ACKNOWLEDGMENTS}

The authors are indebted to R. Fornari for his technical assistance during experiments.

\section{REFERENCES}

[1] K. Balakrishnan, B. Umamaheswari, and K. Latha, Identification of resonance in hybrid stepper motor through measured current dynamics in online for accurate position estimation and control, IEEE Transactions on Industrial Informatics, vol. 9, pp. 1056-1063, 2013.

[2] K.W.-H. Tsui, N.C. Cheung, and K.C.-W. Yuen, Novel modeling and damping technique for hybrid stepper motor, IEEE Transactions on Industrial Electronics, vol. 56, pp. 202-211, 2009.

[3] M. Defoort, F. Nollet, T. Floquet, and W. Perruquetti, A third-order sliding-mode controller for a stepper motor, IEEE Transactions on Industrial Electronics, vol. 56, pp. 3337-3346, 2009.

[4] M. Bendjedia, Y. Ait-Amirat, B. Walther, and A. Berthon, Position control of a sensorless stepper motor, IEEE Transactions on Power Electronics, vol. 27, pp. 578-587, 2012.

[5] Q.N. Le and J.-W. Jeon, Neural-network-based low-speed-damping controller for stepper motor with an FPGA, IEEE Transactions on Industrial Electronics, vol. 57, pp. 3167-3179, 2010.

[6] D. Shin, W. Kim, Y. Lee, and C.C. Chung, Phase-compensated microstepping for permanent-magnet stepper motors, IEEE Transactions on Industrial Electronics, vol. 60, pp. 5773-5780, 2013.

[7] W. Kim, D. Shin, and C.C. Chung, Microstepping with nonlinear torque for permanent magnet stepper motors, IEEE Transactions on Control SYstems Technology, vol. 21, pp. 1971-1979, 2013.

[8] W. Kim, D. Shin, and C.C. Chung, Microstepping using a disturbance observer and a variable structure controller for permanent-magnet stepper motors, IEEE Transactions on Industrial Electronics, vol. 60, pp. 2689-2699, 2013.

[9] M. Butcher, A. Masi, R. Picatoste, and A. Giustiniani, Hybrid stepper motor electrical model extensions for use in intelligent drives, IEEE Transactions on Industrial Electronics, vol. 61, pp. 917-929, 2014.

[10] S. Bifaretti, V. Iacovone, A. Rocchi, P. Tomei, and C.M. Verrelli, Global learning position controls for permanent magnet step motors, IEEE Transactions on Industrial Electronics, vol. 58, pp. 4654-4663, 2011. 
[11] C.M. Verrelli, P. Tomei, V. Salis, and S. Bifaretti, Repetitive learning position control for full order permanent magnet step motors, Automatica, vol. 63, pp. 274-286, 2016.

[12] R. Marino, P. Tomei, and C.M. Verrelli, Robust adaptive learning control for nonlinear systems with extended matching unstructured uncertainties, International Journal of Robust and Nonlinear Control, vol. 22, pp. 645675, 2012.

[13] P. Krishnamurthy and F. Khorrami, Robust adaptive voltage-fed permanent magnet step motor control without current measurements, IEEE Transactions on Control Systems Technology, vol. 11, pp. 415-425, 2003.

[14] S. Li and Z. Liu, Adaptive speed control for permanent-magnet synchronous motor system with variations of load inertia, IEEE Transactions on Industrial Electronics, vol. 56, pp. 3050-3059, 2009.

[15] H. Jin and J. Lee, An RMRAC current regulator for permanent-magnet synchronous motor based on statistical model interpretation, IEEE Transactions on Industrial Electronics, vol. 56, pp. 169-177, 2009.

[16] F. Morel, J.-M. Rétif, X. Lin-Shi, and C. Valentin, Permanent magnet synchronous machine hybrid torque control, IEEE Transactions on Industrial Electronics, vol. 55, pp. 501-511, 2008.

[17] P. Tomei and C.M. Verrelli, Linear repetitive learning controls for nonlinear systems by Padé approximants, International Journal of Adaptive Control and Signal Processing, vol. 29, pp. 783-804, 2015.

[18] C.M. Verrelli, S. Pirozzi, P. Tomei, and C. Natale, Linear repetitive learning controls for robotic manipulators by Padé approximants, IEEE Transactions on Control Systems Technology, vol. 23, pp. 2063-2070, 2015.

[19] S. Bifaretti, P. Tomei, and C.M. Verrelli, A global robust iterative learning position control for current-fed permanent magnet step motors, Automatica, vol. 47, pp. 227-234, 2011.

[20] S. Scalzi, P. Tomei, and C.M. Verrelli, Nonlinear control techniques for the hear rate regulation in treadmill exercises, IEEE Transactions on Biomedical Engineering, vol. 59, pp. 599-603, 2012.

[21] A. Bellini, S. Bifaretti, and S. Costantini, A digital speed filter for motion control drives with a low resolution position encoder, Automatika, vol. 1-2, pp. 67-74, 2003. 